\title{
Mutations in the Antiviral RNAi Defense Pathway Modify Brome mosaic virus RNA Recombinant Profiles
}

\author{
Aleksandra Dzianott, ${ }^{1}$ Joanna Sztuba-Solińska, ${ }^{1}$ and Jozef J. Bujarski ${ }^{1,2}$ \\ ${ }^{1}$ Plant Molecular Biology Center and the Department of Biological Sciences, Northern Illinois University, DeKalb 60115, \\ U.S.A.; ${ }^{2}$ Institute of Bioorganic Chemistry, Polish Academy of Sciences, Noskowskiego 12/14, 61-704 Poznan, Poland
}

Submitted 28 May 2011. Accepted 7 September 2011.

RNA interference (RNAi) mechanism targets viral RNA for degradation. To test whether RNAi gene products contributed to viral RNA recombination, a series of Arabidopsis thaliana RNAi-defective mutants were infected with Brome mosaic virus (BMV) RNAs that have been engineered to support crossovers within the RNA3 segment. Single-cross RNA3-RNA1, RNA3-RNA2, and RNA3-RNA3 recombinants accumulated in both the wild-type (wt) and all knock-out lines at comparable frequencies. However, a reduced accumulation of novel $3^{\prime}$ mosaic RNA3 recombinants was observed in ago1, dcl2, dcl4, and rdr6 lines but not in wt Col-0 or the $d c l 3$ line. A BMV replicase mutant accumulated a low level of RNA3-RNA1 single-cross recombinants in Col-0 plants while, in a $\mathrm{dcl} 2 \mathrm{dcl} 4$ double mutant, the formation of both RNA3-RNA1 and mosaic recombinants was at a low level. A control infection in the cpr5-2 mutant, a more susceptible BMV Arabidopsis host, generated similar-to-Col-0 profiles of both single-cross and mosaic recombinants, indicating that recombinant profiles were, to some extent, independent of a viral replication rate. Also, the relative growth experiments revealed similar selection pressure for recombinants among the host lines. Thus, the altered recombinant RNA profiles have originated at the level of recombinant formation rather than because of altered selection. In conclusion, the viral replicase and the host RNAi gene products contribute in distinct ways to BMV RNA recombination. Our studies reveal that the antiviral RNAi mechanisms are utilized by plant RNA viruses to increase their variability, reminiscent of phenomena previously demonstrated in fungi.

RNA viruses undergo extensive genetic recombination, which provides a means not only for RNA variability or the emergence of new viral strains (Legrand-Abravanel et al. 2007) but also for the repair of RNA replication errors (Elena et al. 2008). Both the RNA template switching by viral replicase (RNA-dependent RNA polymerase [RdRp]) and the rejoining of viral RNA fragments have been implicated in recombination of different RNA viruses (Bujarski 2008a; White and Nagy 2004). In fact, some of them (e.g., Poliovirus spp.) have been shown to utilize both mechanisms to rearrange their progeny RNAs (Agol 2006).

RNA silencing (RNA interference [RNAi]) is an essential cellular defense mechanism operating in eukaryotes to protect

Corresponding author: J. J. Bujarski; Telephone: (815) 753 0601; Fax: (815) 753-7855; E-mail: jbujarski@ niu.edu

* The $\boldsymbol{e}$-Xtra logo stands for "electronic extra" and indicates that two supplementary figures are published online. their genomes against transposons or viruses (Baulcombe 2005; Rosso et al. 2003; Stram and Kuznietzowa 2006; Vaucheret 2006). RNAi is triggered by double-stranded RNAs (dsRNAs), which are processed into small-interfering RNAs (siRNAs) by DICER enzymes (Vazquez 2006). Once formed, the siRNAs guide argonaute (AGO) proteins to complementary single-stranded RNAs (ssRNAs) for cleavage or translational repression (Baulcombe 2005; 2006; Jaskiewicz and Filipowicz 2008; Joshua-Tor 2006; Rana 2007). Cleavage products can be further modified into dsRNAs by cellular RNA-dependent RNA polymerase (RDR), allowing reentry into the cycle leading to an amplification of the degradation process. siRNAs are mobile and can travel long distances to create a gradient of suppression activity. Most plant viruses contain ssRNAs that replicate via dsRNA intermediates. Viral ssRNAs can also be transformed into dsRNAs by cellular RDR. Both forms of viral dsRNA can activate RNAi pathways (Diaz-Pendon et al. 2007, Müller and Imler 2007). Consequently, most RNA viruses counteract the RNAi activity by RNAi suppressors (Llave et al. 2000; Zamore 2004).

Brome mosaic virus (BMV) is a model virus that carries three messenger-sense genomic RNA segments and two subgenomic RNAs (Bujarski 2008b) and is able to infect Arabidopsis thaliana (Dzianott and Bujarski 2004). The previously developed recombination system in Arabidopsis host (Nagy and Bujarski 1993) relies on the formation of heteroduplex between BMV RNA1 and RNA3 (Fig. 1), which allows the nonhomologous RNA3-RNA1 crosses in addition to other nontargeted single-cross BMV RNA recombinants (Dzianott and Bujarski 2004). We refer to single-cross recombinants as those that (formally) emerge due to one cross between RNA molecules. The differences in the recombination patterns observed among different hosts also suggested the role of cellular factors in the modular swap of BMV RNAs (Dzianott and Bujarski 2004; Nagy and Bujarski 1993).

In this work, we have studied whether RNAi gene products contributed to the formation of BMV RNA recombinants. Initially, we show that BMV RNAs are targeted by the RNAi pathway with the aid of dicer-like 2 (DCL2), DCL4, AGO1, and RDR6. Then, ago1, dcl2, dcl3, dcl4, and rdr6 single knock-out mutants and a $d c l 2 d c l 4$ double mutant were inoculated with the recombination system, and types of the progeny recombinant RNA3 were characterized. Mutant hosts $d c l 2$, $d c l 4$, agol, and rdr6 significantly reduced the level of $3^{\prime}$ mosaic recombinants (defined as those that carry the rearranged $3^{\prime}$ untranslated regions [UTR]) but had only a moderate effect on single-cross recombinants. In contrast, the use of a BMV replicase mutant reduced the formation of both single-cross RNA3RNA1 and mosaic recombinant groups in the dcl2 dcl4 host but only RNA3-RNA1 recombinants in the wild-type (wt) Col- 
0 line. A control Arabidopsis mutant (in gene CPR-5 that increased BMV replication) supported both the $3^{\prime}$ mosaic and single-cross recombinants, similar to the observations in Col0 . The relative growth experiments did not reveal differences in the selection pressure among host lines. We conclude that the RNAi-derived host factors affect the formation of BMV RNA recombinants, with a different spectrum of recombinants that are formed through the action of the replicase proteins. Furthermore, our in planta results confirm earlier reports on the roles of DCL2 and AGL2 genes in hypovirus RNA recombination in fungi (Sun et al. 2009).

\section{RESULTS}

Virus accumulation in Arabidopsis RNAi mutant lines.

To test whether BMV is targeted by RNA silencing, Arabidopsis mutant plants impaired in expression of DCL2, DCL4, AGO1, and RDR6 genes were infected with BMV and the level of virus accumulation was determined by dot blot hybridization. The accumulation of BMV RNA was significantly higher in double-mutant line $d c l 2 d c l 4$ (Fig. 2A; Table 1), indicating that BMV was targeted by the RNAi pathway. Similar effects were observed for Cucumber mosaic virus (CMV), which is another Bromoviridae virus (Bouche et al. 2006; Deleris et al. 2006; Morel et al. 2002; Mourrain et al. 2000; Wang et al., 2010). Analogous experiments in the unrelated cpr5-2 knockout mutant showed an approximately fivefold increase in BMV RNA concentration (Table 1), which supported previous observations (Fujisaki et al. 2009).

The BMV virus accumulation in Arabidopsis tissue was also examined by whole-leaf immune-printing (Fig. 2B). The pattern of viral accumulation within the leaves was similar in all the mutant lines, with the virus accumulating within separate patches both at central and more peripheral regions. Again, higher BMV coat protein (CP) concentration was detected in $d c l$-1 $d c l 4-2$ and especially in cpr5-2 plants. No apparent leaf distortion or stunting of the A. thaliana plants was observed after BMV infection.

Virus recombination in Arabidopsis RNAi mutant lines.

A published BMV RNA3 recombination vector named PN8(-) RNA3 (Dzianott and Bujarski 2004; Nagy and Bujarski 1993) was employed in the recombination experiments (Fig. 1). Use of this vector allows all classes of progeny RNA3 recombinants to be assessed, including RNA1-RNA3 heteroduplexmediated and other less-frequent single-cross recombinants, as well as $3^{\prime}$ mosaic recombinants. Below, we describe the effects of gene knockouts on the RNA3 recombinant profiles.

Previously, we have reported that mutations in replicase protein 2a (e.g., the Leu-486 to Phe in mutant DR7) inhibited BMV recombination but resulted in higher titer of the virus (Dzianott et al. 2001; Figlerowicz et al. 1997). Two Arabidopsis lines (wt Col-0 and dcl2-1 dcl4-2) were infected with the DR7 RNA2/PN8(-) RNA3 system. As expected, DR7 virus reached higher titer than the wt virus in both host lines (150 and $300 \%$, respectively) (Table 1).

Also, comparable ratios of unrecombined PN8(-) RNA3 to recombinant progeny RNA3 in view of RNA4 were observed across different lines, as determined by Northern blotting (Fig. $2 \mathrm{C}$, corresponding bands and quantification), which suggested similar selection pressure for BMV RNAs among each of the Arabidopsis host mutant lines.

\section{RNA1-RNA3 recombinants.}

To characterize the recombinants, the $3^{\prime}$ noncoding region of RNA3 was sequenced from 80 cDNA clones containing re-

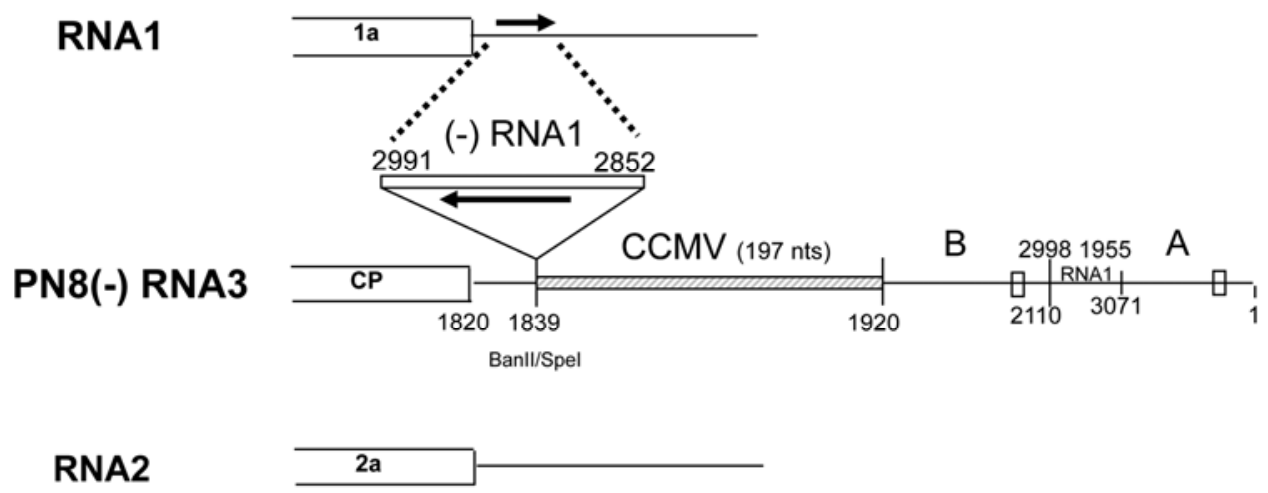

Fig. 1. Schematic representation of the heteroduplex-based Brome mosaic virus (BMV) recombination system. The figure shows both the complete $3^{\prime}$ noncoding regions in all three BMV RNA components (represented by continuous lines) and the downstream portions of the coding regions (represented by open rectangles). The PN8(-) RNA3 recombination vector carries a modified 3' noncoding region that includes a duplication of the 3 ' end sequence (marked as A and B), a Cowpea chlorotic mottle virus RNA3 sequence (marked as CCMV), and the 140-nucleotide antisense BMV RNA1 sequence (with some mismatches) that is complementary to the upstream portion of the $3^{\prime}$ noncoding region in BMV RNA1 (marked as (-) RNA1 within dotted lines) and, thus, forms a heteroduplex structure between RNA3 and -1. Arrows indicate the orientation of the complementary sequences. The nucleotide positions of the RNA sequences are indicated by numbers, both below and above the lines (Nagy and Bujarski 1993 provides a more detailed description of the PN8(-) RNA3 vector). Crossovers occur at the mismatched left portion of the heteroduplex region.

Fig. 2. Accumulation of Brome mosaic virus (BMV) and BMV RNA in wild-type (wt) and mutant Arabidopsis lines. A, Time course of the accumulation of BMV RNA in the aerial tissue from Arabidopsis seedlings. Plants were inoculated with BMV wt RNA1 and RNA2 and PN8(-) RNA3 followed by extraction of total RNA at the indicated time points postinoculation. The RNA concentration was determined by using the quantitative dot blot hybridization. B, Pressblot analysis of BMV multiplication. Whole plants were pressed into chromatography papers at 14 days postinoculation and viral coat protein (CP) was detected immunologically, using anti-BMV antiserum. C, Northern blot analysis of BMV RNA in total RNA extracts from plants infected with wt RNA1 and RNA2 and PN8(-) RNA3. After extraction, the RNA was separated by electrophoresis in a 0.9\% agarose/formamide-formaldehyde gel (two runs per each infected host line, marked by numerals 1 and 2), blotted, and probed with a radioactive RNA probe that was complementary to the $3^{\prime} 200$ nucleotides in the (+) strand. Names of Arabidopsis mutants are shown on top while the positions of individual BMV RNAs are indicated on the right-hand side. The concentration of total RNA from cpr5-2 was diluted 10-fold prior of electrophoresis. The quantification bars (as average from two separate runs 1 and 2) for either unrecombined PN8(-) RNA3, recombinant RNA3, or RNA4 bands are shown below each host line. 


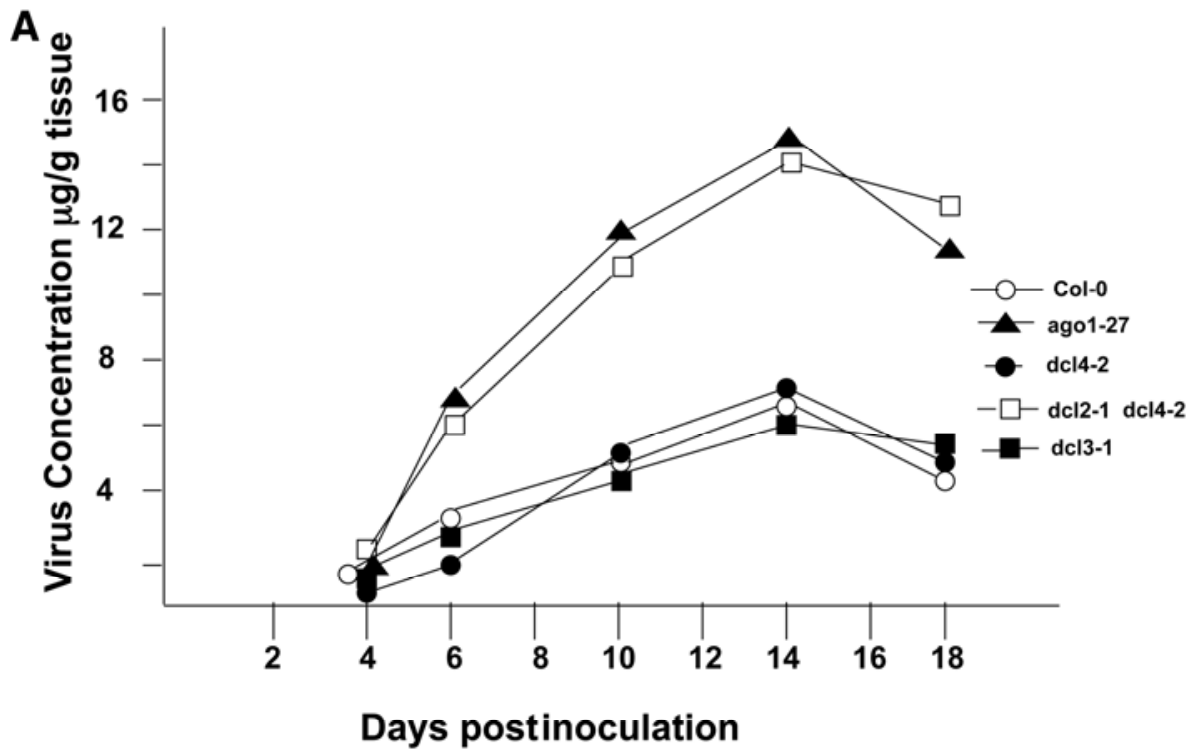

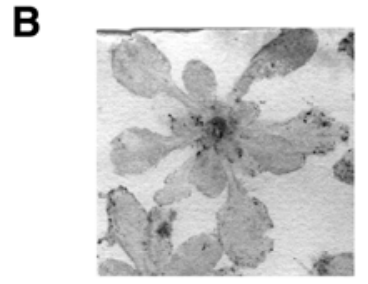

Col-0

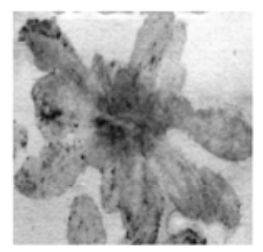

$d c / 2-1 \times d c / 4-2$

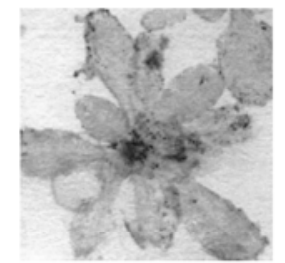

$d c / 2-1$

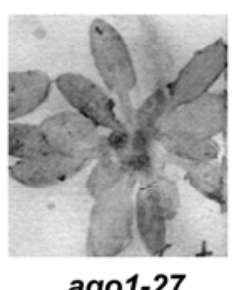

ago1-27

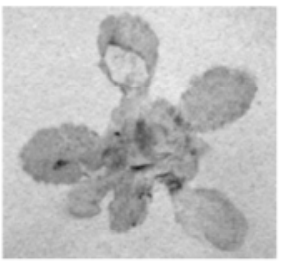

dc/3-1

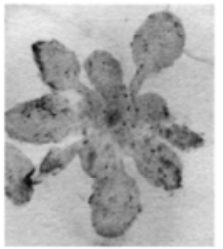

rdr6

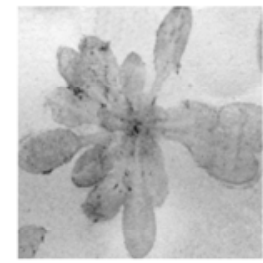

$d c / 4-2$

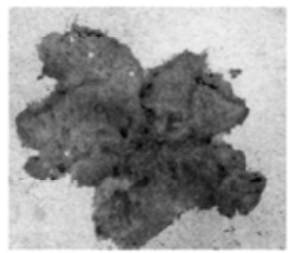

cpr5-2

C

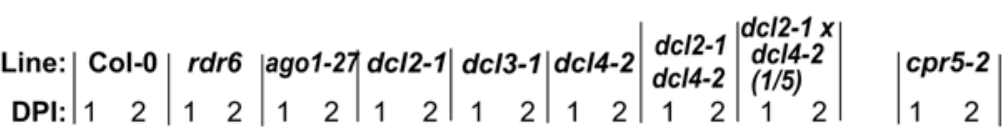
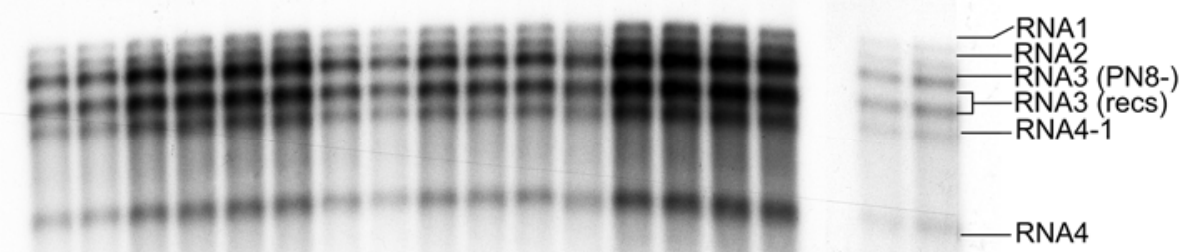

Percent of

Total Density

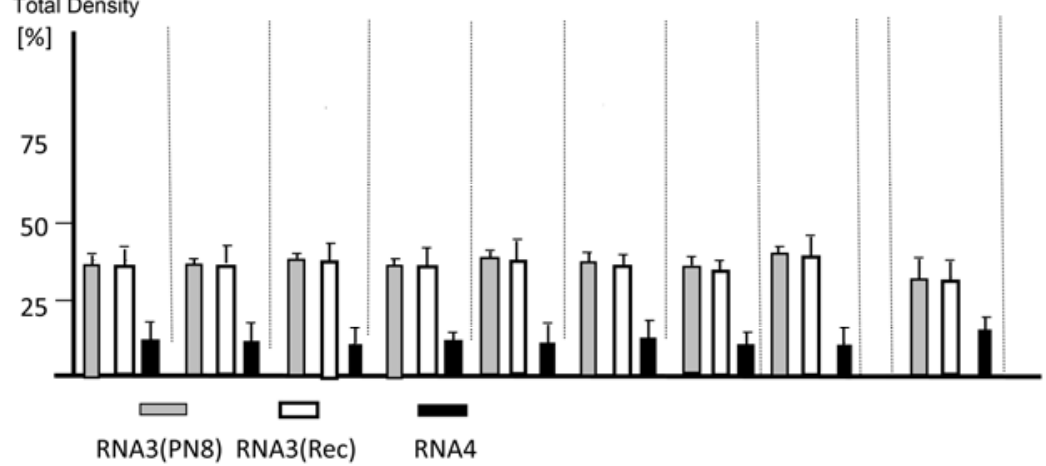


combinant variants per each line. Among four classes of RNA3 recombinants (Table 1), the targeted RNA1-RNA3 recombinants (Fig. 3) accumulated at high levels, ranging between 70 and $80 \%$ in dcl2-1, dcl4-2, dcl2-1 dcl4-2, agol-27, $r d r 6$, or $c p r 5-2$, and between 50 and $60 \%$ in wt Col-0 and dcl3-1 lines. When the DR7 BMV was used, the fraction of RNA1-RNA3 recombinants was significantly reduced in both Col-0 and $d c l 2-1 d c l 4-2$ (to 5\%), thus confirming the previous observations that DR7 mutation inhibited the heteroduplexmediated template switching (Dzianott et al. 2001; Figlerowicz et al. 1997; Nagy and Bujarski 1993).

The average end points on the RNA3 and RNA1 templates and their standard deviations were calculated and compared. For all Arabidopsis lines, the crossovers occurred at the left edge of the heteroduplex region (Fig. 3). In Col-0, dcl3-1, and cpr5-2 plants, the end points of crossover sites were shifted by a 20- to 30-nucleotide (nt) gap. In the remaining mutants, the end points were nearly symmetrical whereas, in agol-27, the end points overlapped. Interestingly, the end points in $r d r 6$ and dcl3-1 were as in Col-0 but they occurred in an opposite arrangement in $d c l 2-1, d c l 4-2$, and $d c l 2-1 d c l 4-2$, as reported previously for Chenopodium quinoa (Dzianott and Bujarski 2004). Thus, the specificity of DCL2 and DCL4 host factors might differently govern BMV recombination crossovers. Overall, the RNAi gene knockouts reduced the asymmetry of end points versus Col-0, except in $d c l 3-1$ and cpr5-2 (discussed below).

\section{RNA3-RNA2 and RNA3-RNA3 recombinants.}

RNA2-RNA3 and RNA3-RNA3 recombinants accumulated at low frequency in the presence of wt protein $2 \mathrm{a}$, whereas DR7 2a generated a higher fraction of both types (Table 1). These recombinants were not reported before for the PN8(-) RNA3 vector but similar crosses were described for the homologous recombination vector PN-H26 RNA3 (Nagy et al. 1999; Olsthoorn et al. 2002). The RNA3-RNA2 crosses clustered at $3^{\prime}$ noncoding sequences predicted to both form an imperfect heteroduplex and display a significant level of homology within the RNA3-RNA1 insert/junction region of PN8(-)
RNA3 and a region between nucleotides 2,540 and 2,570 in wt RNA2 (Supplementary Fig. S1), This suggests that the complementarity- or the homology-based replicase-switching mechanisms might support the formation of RNA3-RNA2 recombinants (discussed below).

The group of recombinants formed among just PN8(-) RNA3 molecules was identified in all experiments. The junctions clustered within the internal RNA3-RNA1 region (nucleotide 1,839) and the $3^{\prime}$ region $A$ (nucleotides 1,955 to 3,071 ) of the PN8(-) RNA3 construct (Supplementary Fig. S2S). Similar to RNA3-RNA2 crosses, these sequences displayed some degree of both homology and complementarity to each other. The recombinants carried either the parental $3^{\prime}$ terminal 20-nt deletion (in region A) or had this deletion repaired via secondary crosses with RNA1 or RNA2 3' ends (not specified). RNA3-RNA3 recombinants were somewhat more frequent than RNA3-RNA2 recombinants, especially in DR7-infected plants, perhaps due to reduced competition with the lowered RNA1-RNA3 recombinants (discussed below).

\section{$3^{\prime}$ mosaic recombinants.}

A novel group of 3' rearranged (mosaic or chimeric) RNA3 recombinants was identified (Fig. 4). For Col-0 plants, the mosaic recombinants carried either the 3 ' region of the PN8(-) RNA3 vector (e.g., clone number 44), or were more rearranged with the RNA1 3' region (clone number 48), RNA3 sequences of negative polarity (clone numbers 59 and 65), the inner RNA3 region (clone number 58), or a 3' UTR of RNA1 ligated downstream of the $\mathrm{CP}$ open reading frame (ORF) (clone numbers 61 and 62). Apparently, these reshufflings occurred via multiple recombination events. None of the recombinants carried the parental (-) RNA1 insert of the PN8(-) RNA3 vector (at nucleotide position 1,839).

The mosaic recombinants represented $35 \%$ of the entire recombinant pool in wt Col-0 host and $25 \%$ in $d c l 3-1$ plants but only 5, 10, and 10\% in ago 1-27, $d c l 2-1$, and $d c l 4-2$ plants, respectively. None of the mosaic recombinants were found in rdr6 and dcl2-1 dcl4-2 plants (Table 1; Fig. 4). Also, for the DR7 mutant, the Col-0 plants accumulated $35 \%$ mosaic re-

Table 1. Accumulation of classes of Brome mosaic virus (BMV) RNA3 recombinants in Arabidopsis lines by using the PN8(-) RNA3 recombination system

\begin{tabular}{|c|c|c|c|c|c|}
\hline \multirow[b]{3}{*}{ Mutant ${ }^{b}$} & \multirow[b]{3}{*}{ Accumulation $(\%)^{\mathrm{c}}$} & \multicolumn{4}{|c|}{ Recombinant RNA3 class $^{\mathrm{a}}$} \\
\hline & & \multicolumn{3}{|c|}{ Single-cross recombinants } & \multirow[b]{2}{*}{ "Chimeric" recombinants } \\
\hline & & RNA3-RNA1 $^{\text {d }}$ & RNA3-RNA2 ${ }^{\mathrm{e}}$ & RNA3-RNA3 $^{\text {f }}$ & \\
\hline \multicolumn{6}{|l|}{ wt RNA2h } \\
\hline Col-0 & 100 & $50 \pm 5$ & $5 \pm .5$ & $10 \pm 1$ & $35 \pm 3$ \\
\hline Col-0 (1/5) & 85 & $55 \pm 5$ & $5 \pm .5$ & $10 \pm 1$ & $30 \pm 4$ \\
\hline$r d r 6(\mathrm{RdRp})$ & 200 & $80 \pm 6$ & $5 \pm .5$ & $15 \pm 2$ & 0 \\
\hline agol-27 & 250 & $70 \pm 5$ & $10 \pm 1$ & $15 \pm 1$ & $5 \pm 2$ \\
\hline dcl2-1 & 90 & $70 \pm 5$ & $10 \pm 2$ & $10 \pm 1$ & $10 \pm 2$ \\
\hline$d c l 3-1$ & 95 & $60 \pm 4$ & $5 \pm .5$ & $10 \pm 1$ & $25 \pm 4$ \\
\hline$d c l 4-2$ & 110 & $75 \pm 6$ & $5 \pm 1$ & $10 \pm .5$ & $10 \pm 1$ \\
\hline$d c l 2-1 d c l 4-2$ & 250 & $75 \pm 5$ & $5 \pm .5$ & $20 \pm 3$ & 0 \\
\hline dcl2-1 dcl4-2 (1/5) & 180 & $65 \pm 4$ & $10 \pm 2$ & $25 \pm 4$ & 0 \\
\hline $\operatorname{cpr} 5-2$ & 550 & $75 \pm 7$ & $5 \pm .5$ & $5 \pm 2$ & $20 \pm 3$ \\
\hline \multicolumn{6}{|l|}{ DR7 RNA2 ${ }^{\mathrm{i}}$} \\
\hline $\mathrm{Col}-0$ & 150 & $5 \pm .5$ & $25 \pm 2$ & $35 \pm 4$ & $35 \pm 4$ \\
\hline$d c l 2-1$ dcl4-2 & 300 & $5 \pm .5$ & $35 \pm 2$ & $55 \pm 6$ & $5 \pm 3$ \\
\hline
\end{tabular}

${ }^{\mathrm{a}}$ Based on sequencing of 80 cDNA clones of recombinant sequence per each Arabidopsis mutant line.

${ }^{\mathrm{b}}$ Names of the Arabidopsis mutant lines tested. In the case of Col-0 and $d c l 2-1 d c l 4-2$, two inoculation experiments were done, using either the regular (RNAs 1, 2, and 3 at $5 \mu \mathrm{g}$ each per five leaves) or reduced by $80 \%$ (marked as "1/5") amount of the separately in vitro-transcribed BMV RNAs.

${ }^{\mathrm{c}}$ BMV RNA accumulation compared with wild-type (wt) Col-0 14 days postinoculation. All figures represent average from three to five infected plants.

${ }^{\mathrm{d}}$ Heteroduplex-targeted RNA3-RNA1 recombinants.

${ }^{e}$ Recombinants nontargeted by the RNA3-RNA1 heteroduplex due to crosses between PN8(-) RNA3 and wt RNA2.

${ }^{\mathrm{f}}$ Recombinants nontargeted by the RNA3-RNA1 heteroduplex due to crosses among the molecules of PN8(-) RNA3.

g These RNA3 recombinants carry the mosaic 3 ' noncoding region due to crosses among different BMV RNAs.

${ }^{\mathrm{h}} \mathrm{wt}$ BMV 2a protein.

${ }^{i}$ Mutation Leu486 to Phe in BMV 2a open reading frame. 
combinants but the $d c l 2-1 d c l 4-2$ line supported only $5 \%$ of mosaic recombinants. These results implied that the mosaic recombinants arose as a consequence of RNAi pathway rather than by the "classical" BMV replicase template switching mechanism. Mutant cpr5-2 accumulated $20 \%$ of mosaic recombinants, demonstrating that the CPR5 gene was not essential for the formation of mosaic recombinants.

To determine whether the amount of RNA inoculum affected the mosaic recombinants, both Col- 0 and $d c l 2-1 d c l 4-2$ plants were inoculated with a reduced RNA concentration $(1 \mu \mathrm{g}$ of each RNA component per five leaves). Similar to the higher concentration, a significant fraction of mosaic recombinants accumulated in Col-0 but not in $d c l 2-1 d c l 4-2$ plants (Table 1). This implied that degradation or prior RNA recombination in the inoculum were not the major factors for mosaic recombinants.

The types of mosaic recombinants isolated from Arabidopsis mutant lines were similar to those from Col-0, likely reflecting similar mechanisms of their generation and compara- ble selection pressure for replicable variants. The junctions occurred within short nucleotide regions (Fig. 4, black bars), suggesting limited randomness during crossover events. As in Col-0, the mosaic recombinants accumulated mainly in lines carrying functional DCL2 and DCL4 RNAi genes (e.g., in Col-0, dcl3-1, or cpr5-2) (Table 1), confirming the involvement of the corresponding host RNAi factors (discussed below).

\section{Growth rates of mosaic recombinants.}

The relative concentration of recombined and unrecombined BMV RNA3 components remained similar among the host mutants (Fig. 2C), suggesting comparable selection pressure. To further test whether selection affected recombinant profiles, two full-length mosaic RNA3 variants of different size (clone number 44 carried longer sequence than clone number 58) (Fig. 4) were reconstructed and co-inoculated (plus wt RNAs 1 and 2) on selected mutants (dcl2-1 dcl4-2, dcl3-1, dcl4-2,

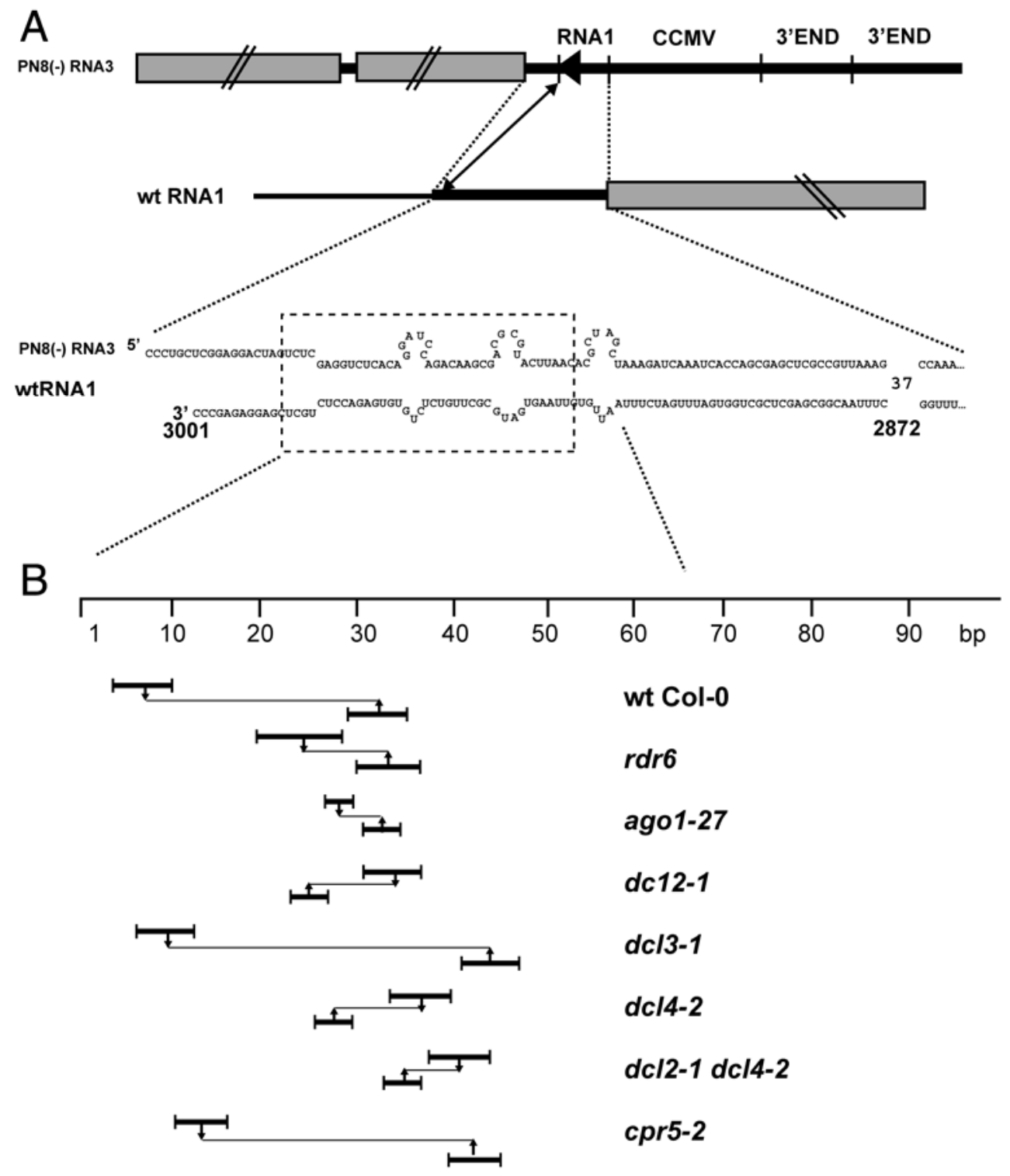

Fig. 3. Characterization of the RNA3-RNA1 recombinants at the heteroduplex region. A, Diagram summarizing the location of the RNA3-RNA1 cross-sites. Nucleotide sequences forming the heteroduplex region are displayed for both the PN8(-) RNA3 and the wild-type (wt) RNA1 sequences. The region of crossovers is depicted by a thick double arrow and is encased by dotted lines on the left side of the sequences. B, Location of mean positions of crossover sites in the recombinants from different Arabidopsis lines. Cross sites are shown by pairs of connected arrowheads facing down and up, representing RNA3 and RNA1 sequences, respectively. Horizontal bars at each arrowhead indicate standard deviation from the mean for each distribution. The top scale indicates the position of base pairs along the heteroduplex region, counting from the first base-pair (G-C) on the left-hand side. 
agol-27, and wt Col-0). The time course of accumulation of both RNA3 variants was determined by comparing the intensity of corresponding bands in Northern blots (not shown), after probing with a $3 \mathrm{a}$ ORF RNA3-specific probe of total RNA extracts at $6,8,10,12$, and 14 days postinoculation. The ratio of recombinant numbers 44 to 58 decreased over the infection period to a similar degree in different mutant lines, reflecting an equivalent selection pressure (Fig. 5). Thus, the differences in the recombinant profiles likely originated at the level of recombinant formation rather than because of different selection.

\section{DISCUSSION}

Replicase-driven template switching has been reported as the mechanism of genetic recombination in RNA viruses (Chao 2007; Hanson et al. 2005), including BMV (Alejska et al. 2005; Olsthoorn et al. 2002; Urbanowicz et al. 2005), with host factors being implicated in affecting the final pattern of BMV RNA recombinants (Dzianott and Bujarski 2004). Here, we demonstrate that some of the BMV RNA recombinants can arise due to RNAi activity, in a mechanism distinct from the template-switching process. Thus, the otherwise essential cellular defense antiviral mechanism (Dunoyer and Voinnet 2005) also contributes to the variability of the BMV RNA genome.
Single-cross RNA3 recombinants.

In previous studies (Dzianott and Bujarski 2004), we have shown that PN8(-) RNA3 directed the RNA1/RNA3 recombination in Col-0 Arabidopsis whereas, in C. quinoa, the crossover sites were shifted at more inner positions within the heteroduplex (Nagy and Bujarski 1993). Here, we demonstrated that the mean location of crossovers (Fig. 3) was not affected by the RNAi mutants tested in this work, suggesting the independence of the mechanism upon the RNAi host factors. The formation of RNA3-RNA1 recombinants was inhibited by the DR7 BMV replicase mutant (Table 1), already demonstrated by us in C. quinoa (Nagy et al. 1995). Therefore, the replicasedriven template switch (Chetverin et al. 2005; LegrandAbravanel et al. 2007; Llave et al. 2000) must be responsible for the formation of RNA1-RNA3 recombinants. The observed differences in the arrangement of donor and acceptor end points (Fig. 3) might be because the knock-out of RDR6, AGO1, or DCL could alter the activity of BMV RdRp or the structured regions of the RNAs, which then might enable DICER contribution to the position of donor and acceptor end points. Also, the viral RNAs derived from the activity of RNAi might affect the switching properties of the replicase (Nagy et al. 1995). The observed clustering of RNA3-RNA2 and RNA3-RNA3 recombinants within a region that displayed some degree of both homology and complementarity might be

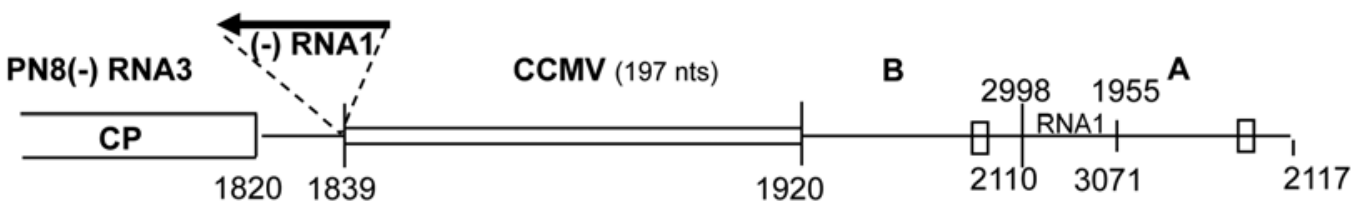

Col-0 rdr-6 ago1-27 dc/2-1 dc/3-1 dc/4-2 dc/2 $x$ dc/4

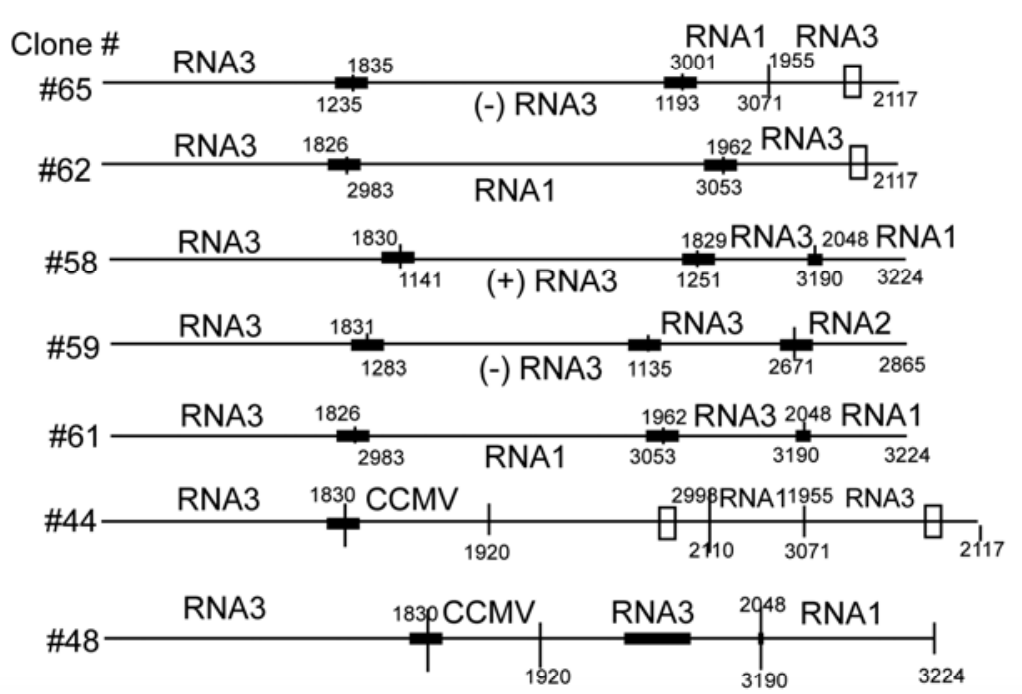

$\begin{array}{lllllll}2 & 0 & 0 & 1 & 4 & 0 & 0 \\ 4 & 0 & 2 & 0 & 0 & 1 & 0 \\ 4 & 0 & 0 & 1 & 3 & 2 & 0 \\ 2 & 0 & 0 & 0 & 0 & 0 & 0 \\ 5 & 0 & 2 & 3 & 3 & 0 & 0 \\ 5 & 0 & 0 & 2 & 9 & 3 & 0\end{array}$
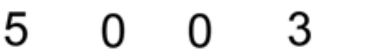

$\begin{array}{lll}3 & 4 & 0\end{array}$

\section{Total \# of identified "mosaic" clones: $\begin{array}{lllllll}27 & 0 & 4 & 10 & 22 & 10 & 0\end{array}$ \% of total \# of analyzed recombinant clones: $\begin{array}{lllllll}34 & 0 & 5 & 8 & 27 & 8 & 0\end{array}$}

Fig. 4. Characterization of RNA3 mosaic recombinants. Top: diagram of the $3^{\prime}$ portion of PN8(-) RNA3 molecule. Bottom: schematic representation of individual types of mosaic recombinants that were identified in wild-type and mutant Arabidopsis lines. Numerals on the left refer to prototypical cDNA clones found in Col-0 plants. The long horizontal lines represent the $3^{\prime}$ noncoding region (not to scale), and the nucleotide junctions between different Brome mosaic virus (BMV) RNA fragments are specified by nucleotide positions below and above a short vertical line at each junction. Black bars on the lines mark the 5- to 10-nucleotide range of the junction sites, as mapped in recombinants isolated from mutant Arabidopsis lines. The table on the right summarizes the numbers of mosaic and their percentage of total recombinant cDNA RNA3 clones that were characterized per each host line (specified on the top). $\mathrm{CP}=\mathrm{coat}$ protein; CCMV = Cowpea chlorotic mottle virus. 
because the complementarity could aid the heteroduplexdriven switches (Nagy et al. 1999), whereas the homology could support the primer-extension reaction (Alejska et al. 2005). It is unclear whether the above three recombinant classes were generated by the same mechanism because, in contrast to the inhibition of RNA3-RNA1 recombinants, DR7 mutation enhanced the formation of the latter two groups (Table 1). More data are required to determine the exact mechanisms.

\section{Chimeric RNA3 recombinants.}

Significantly fewer chimeric (mosaic) recombinants were detected in RNAi mutants than in Col- 0 or in an unrelated mutant (Table 1; Fig. 4). DCL2 and DCL4 both act as RNAi silencers for CMV and other viruses (Bouche et al. 2006; Deleris et al. 2006; Ding and Voinnet 2007; Fusaro et al. 2006), whereas DCL3 has no defined contribution to antiviral silencing. In that respect, our results support these data. Also, for the DR7 BMV mutant, the Col-0 plants accrued $35 \%$ mosaic recombinants but the $d c 2-1 d c l 4-2$ line supported only $5 \%$. This suggests that the formation of mosaic recombinants is mainly aided by RNAi activity rather than exclusively by BMV replicase.

The RNAi mutant analysis also provided information regarding the RNA pools used for viral recombination. Because dsRNAs (either structured ssRNA or replication intermediates) dice into primary siRNAs, and cleavage fragments are copied by RDR6 and then diced again into secondary siRNAs (Chapman and Carrington 2007), we surmise that the incompletely diced RNA fragments may be employed as building blocks for BMV recombination swap. However, without further experimental evidence, no definite conclusions can be drawn. It could, for example, be that the pool of viral RNAs available for recombination changes qualitatively in different RNAi Arabidopsis mutants. It has previously been shown that the sense and antisense strands of plant RNA viruses are differentially targeted by sRNAs (Donaire et al. 2009). Thus, by mutating certain RNA-silencing components, the ratio of sense and antisense strands might conceivably change.

Also, DCL2 and DCL4 (e.g., in wt plants or in $d c l 3-1$ ) could generate BMV RNA fragments at structured regions (Hamilton and Baulcombe 1999; Molnar et al. 2005), such as the $3^{\prime}$ tRNA-like structure or the heteroduplex region (Fig. 1). AGO1 as well as DCL2 and DCL4 act in both production and assembly of primary and secondary siRNAs and, thus, the analysis of agol-27, dcl2-1, and dcl4-2 plants cannot tell us which RNA fraction is used for mosaic recombinants. In contrast, RDR6 operates during production of dsRNAs from viral RNA cleavage products. Because no mosaic recombinants were found in $r d r 6$ plants, the RNAs derived from the RDR6 activity (such as the long antisense RNA or long dsRNA) are likely used as templates to generate mosaic recombinants.

The RNAi-derived fragments might further degrade via mRNA decay mechanisms, generating a variety of RNA fragments (Gy et al. 2007; Souret et al. 2004). In two mosaic clones (numbers 59 and 65), the detected presence of minussense RNA3 inner fragments could form local ds regions providing new targets for further dicing.

Mosaic RNA3 recombinants could arise by rejoining of RNAi-generated fragments, via either end-to-end template switching (Cheng et al. 2006), trans-esterification, or religation aided by host ligases as proposed for, for example, Tomato bushy stunt virus RNA recombination (Cogoni and Macino 1999). The end-to-end process has been reported in vitro for BMV (Kim and Kao 2001), and in vivo for Poliovirus spp. (Gmyl et al. 2003) or Q-beta RNA phage (Agol 2006). The selection pressure for more fit recombinants may contribute, as well, and it might be host dependent. For instance, the mosaic RNA3 recombinants were not reported in barley, Nicotiana benthamiana, or $C$. quinoa, the efficient BMV hosts. We speculate that a generally inefficient BMV accumulation in Arabidopsis reduces the competition for the best fit variants, allowing the accumulation of mosaic recombinants at detectable levels.

The rdr6 and agol mutations enhance the susceptibility to CMV (Ding and Voinnet 2007), likely by limiting the spread of RNAi signals and reducing RNA cleavage. Additionally, the CMV 2b suppressor targets the AGO1 protein (Zhang et al. 2006). The increased viral titer in rdr6 and agol mutants was observed for BMV (Table 1). This makes us speculate (but requires experimental confirmation) that a yet-to-be-identified BMV suppressor targets AGO1 protein in Col-0 but not in agol-27 plants, affecting the production of mosaic recombinants.

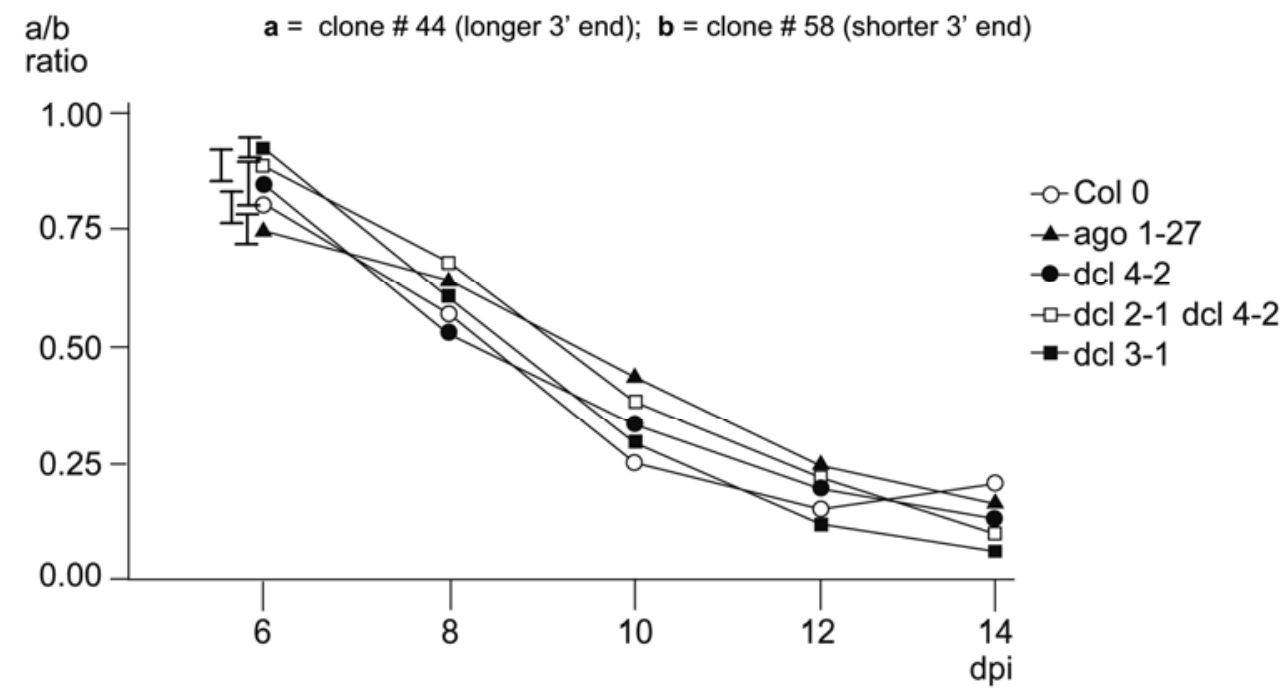

Fig. 5. Determination of selection pressure for Brome mosaic virus (BMV) RNA3 mosaic recombinants. The graph shows changes in the ratio of accumulation of two reconstructed parental mosaic RNA3 recombinants (clones 44 ["a", longer one] and 58 ["b", shorter one] [plus wild-type RNA1 and RNA2]) after co-infection in specified Arabidopsis lines. The time course of accumulation of both variants was quantified based on the intensity of separate bands (size difference between a and $b=261$ nucleotides) in Northern blots. Each concentration point represents an average from four inoculated seedlings. Standard deviation bars are shown as short vertical lines only for day 6 (on the left hand side) for better picture clarity (at other day points, the error values were similar); dpi = days postinoculation. 
Effect of cpr5-2.

CPR5 mutants have pleiotropic effects on defense-related genes and cause growth defects in Arabidopsis. Fijusaki and associates (2009) have observed that the loss of function of CPR (cpr5-2) enhanced BMV multiplication, suggesting that cpr5-2 eliminates anti-BMV resistance, independent of the expression of BMV replicase or on RNAi pathways. Our data confirm these results because we observed confined viral spread within the leaf tissue in all but cpr5-2 variants (Fig. 2B). Only two kinds of recombinants, the targeted RNA3RNA1 crosses and the mosaic recombinants, were identified in cpr5-2 (Table 1). If, indeed, the mechanism of cpr5-2 stimulation is independent of replicase expression and RNAi gene products, the observed effects might be due to increased selection in a more efficient host. However, it is not known whether RNAi-generated BMV RNA fragments survive longer time in cpr5-2.

In this preliminary report, the effects of DCL1 knockouts were not tested. DCL1 generates micro-RNAs (miRNAs) but it can facilitate functions of other DCL proteins (Blevins et al. 2006; Dunoyer et al. 2010) or even process dsRNAs in the absence of other DCL genes (Bouche et al. 2006; Zhang et al. 2007) and, thus, contribute to the production of BMV recombinants.

The general mechanism of RNA recombination is expected to be universal among RNA viruses, suggesting a widespread contribution of RNAi in rearrangement of viral RNAs. The evidence for the role of RNA silencing as an antiviral defense in fungi was provided by Segers and associates (2007), followed by the reported role of $a g l-2$ and $d c l 2-1$ in hypovirus RNA recombination (Sun et al. 2009; Zhang and Nuss 2008). Evidently, RNA viruses can employ the antiviral RNAi pathways for enhancing their own genome variability, boosting the viral genetic shift (Duffy et al. 2008; Elena et al. 2008; Escarmís et al. 2006). Our results provide innovate means to examine the dynamics of the plant viral RNA recombinant pattern and the various mechanisms that might shape it.

\section{MATERIALS AND METHODS}

\section{Plant and virus materials.}

All mutants belong to the A. thaliana Col-0 ecotype and have been described before (Alonso et al. 2003; Morel et al. 2002; Mourrain et al. 2000; Rosso et al. 2003; Xie et al. 2005); dcl2-1, dcl3-1, dcl4-2, and dcl2-1 dcl4-2 were provided by J. Carrington; rdr6 (sgs2-1) and agol-27 were provided by $\mathrm{H}$. Vaucheret; whereas cpr5-2 was purchased from the Arabidopsis Biological Resource Center (The Ohio State University, Columbus, OH, U.S.A.).

The full-length cDNA clones, corresponding to wt BMV RNAs 1, 2, and 3, type (Russian) strain, were obtained from the P. Ahlquist laboratory as plasmids pB1TP3, pB2TP5, and pB3TP7, respectively (Janda et al. 1987). The BMV PN8(-) RNA3 recombination vector was transcribed from plasmid pPN8(-) RNA3, as described (Nagy and Bujarski 1993). Plasmid pB2DR7 (a gift from P. Ahlquist) (Kroner et al. 1990) was used to synthesize BMV RNA2 transcripts bearing the DR7 mutation (Leu486-Phe) in 2 a protein.

\section{In vitro transcription, plant inoculations, and virus infection.}

In order to increase the stability of BMV RNA components during infection (especially the progeny RNA3 recombinants and subgenomic RNAs due to their expected limited co-packaging with RNA3), the infected seedlings were incubated at a lower temperature, and total RNA was analyzed at a shorter time ( 9 days) postinfection. Thus, the seedlings of $A$. thaliana lines were grown in a controlled-environment chamber under the following conditions: $150 \mathrm{mE} / \mathrm{m}^{2} / \mathrm{s}$ irradiance, $8 \mathrm{~h}$ of light, $16^{\circ} \mathrm{C}$ night temperature, and $18^{\circ} \mathrm{C}$ day temperature. Three leaves were inoculated with a mixture of transcribed wt and mutated BMV RNAs that were synthesized by run-off in vitro transcription with the MEGAscript kit from Ambion (Austin, TX, U.S.A.), as described (Dzianott and Bujarski 2004). The integrity of the RNA products was confirmed by electrophoresis in denaturing agarose gels.

\section{Tissue imprinting assay.}

Tissue printings were performed as described (Takahashi et al. 2002). Briefly, the 14- to 18-day-old (five- to six-leaf stage) seedlings of $A$. thaliana lines were inoculated with the transcribed BMV RNA recombination system at $100 \mu \mathrm{g} \mathrm{ml}^{-1}$, and the plants were incubated for 14 days in a growth chamber. Whole Arabidopsis plants were sandwiched between filter papers and pressed, plant debris were removed, and the blotted paper was gently shaken or washed with a consecutive series of the following solutions: i) Triton $\mathrm{X}-100 ; 10 \mathrm{mM}$ sodium phosphate buffer $(\mathrm{pH} 7.2)$ containing $0.9 \% \mathrm{NaCl}, 0.1 \%$ Tween 20 , and 3\% skim milk (phosphate-buffered saline-Tween [PBST]); ii) rabbit antiserum to BMV-R CP (Agdia, Elkhart, IN, U.S.A.); iii) PBST; iv) goat anti-rabbit immunoglobulin G-alkaline phosphatase conjugate (BioRad, Hercules, CA, U.S.A.); v) PBST; and vi) $100 \mathrm{mM}$ Tris- $\mathrm{HCl}(\mathrm{pH} 9.5), 100 \mathrm{mM} \mathrm{NaCl}$, and $5 \mathrm{mM}$ $\mathrm{MgCl}_{2}$. At the end, the paper was incubated with the developer for $20 \mathrm{~min}$ and the staining reaction was stopped with distilled water. The blots were dried and scanned.

\section{RNA analyses.}

For Northern blotting, the total RNA extracts were separated by electrophoresis in a denaturing formaldehyde/formamide agarose gel, followed by blotting to the Hybond-N+ nylon membrane (Amersham-Pharmacia Biotech, Piscataway, NJ, U.S.A.), and probing with a $200-n t$ radioactive transcript that was complementary to the 3 ' region of BMV RNAs (Kim and Kao 2001). The gels were exposed to X-ray film, scanned, and subjected to the computer-based densitometry by using an ImageQuant 5.0 program from Amersham-Biosciences (Piscataway, NJ, U.S.A.).

\section{cDNA cloning and sequencing of progeny BMV RNA3 recombinants.}

Aerial parts of 2 to 10 plants were harvested and total RNA was extracted, as described previously (Dzianott and Bujarski 2004). To generate cDNA clones of BMV RNA recombinants, the RNA3 sequences were amplified with reverse-transcription polymerase chain reaction (RT-PCR) (Bruyere et al. 2000) by using TaqI polymerase and a pair of the oligonucleotides: AB75 (5'-CAGTGAATTCTGGTCTCTTTTAGAGATTTACA GTG-3'), that was complementary to $3^{\prime}$ terminal nucleotide 2,117 to 2,093 of wt RNA3 (EcoRI site underlined), and JJB-2 (5'-GAAGCAGTGCCTGCTAAGGCGGTC-3'), that represented the nucleotides 1,731 to 1,754 of wt RNA3. The resulting cDNA products were separated by electrophoresis in agarose gel, the bands were cut out from the gel, and the products were purified on QIAquick spin columns (Qiagen Inc.; catalog number 28704). Then, the DNA was digested with terminal restriction enzymes and ligated between these sites in the pUC18 cloning vector, and the resulting $3^{\prime}$ RNA3 cDNA clones ( 80 clones that carried recombined sequences per each Arabidopsis mutant) were sequenced by using primers complementary to either flanking vector sides. This allowed us to map the crossover sites within the recombinant RNA3 3' UTR.

Control RT-PCR reactions were performed (Nagy et al. 1995). In one reaction, the in vitro transcribed BMV RNAs 
were amplified as cDNA followed by cloning. This revealed only the unrecombined PN8(-) RNA3 sequences (data not shown). In another experiment, RT-PCR reactions were repeated twice on the same total RNA extracts from BMVinfected Arabidopsis plants, and this reproducibly amplified similar pattern of cDNA products (data not shown). Thus, the identified RNA3 recombinants were not the RT-PCR artifacts.

\section{Reconstruction of full-length $3^{\prime}$ mosaic RNA3 recombinants.}

Two full-length mosaic RNA3 recombinants were reconstructed from the $3^{\prime}$ mosaic RNA3 recombinant cDNA clones 44 and 58 (Fig. 4). The corresponding wt $3^{\prime}$ noncoding sequence in pB3TP7 plasmid was replaced between the XbaI and EcoRI restriction sites with the $3^{\prime}$ mosaic region and the resulting full-length cDNA constructs were linearized at the $3^{\prime}$ end by restriction enzyme digestion followed by the run-off in vitro transcription.

\section{Statistical analysis.}

To test whether the observed shifts of crossovers (Fig. 3) were statistically significant, the mean recombination sites and the standard deviation from that mean were calculated as published before (Nagy et al. 1995). Briefly, the mean location on the heteroduplex region was calculated with the SuperANOVA program by using the nucleotide position of each crossover site in all the clones. To identify which of the means differed significantly from any others, the Duncan new multiple-range option in SuperANOVA was applied (Winer 1962). Duncan analysis allowed us to compute critical ranges.

\section{ACKNOWLEDGMENTS}

We thank S. Hill, D. Nuss, and H. Vaucheret for comments and discussions; A. Simon, for suggesting the use of BMV replicase mutants in recombination experiments; and W. Buikema at the University of Chicago CRC DNA Sequencing and Genotyping Facility and S. Grayburn at Northern Illinois University DNA Core Facility of Plant Molecular Biology Center for sequencing of cDNA clones. This work was supported by grants from National Science Foundation (MCB-0920617), National Institutes of Health (G1A62203), and the Plant Molecular Biology Center at Northern Illinois University.

\section{LITERATURE CITED}

Agol, V. I. 2006. Molecular mechanisms of poliovirus variation and evolution. Curr. Top. Microbiol. Immunol. 299:211-259.

Alejska, M., Malinowska, N., Urbanowicz, A., and Figlerowicz, M. 2005. Two types of non-homologous RNA recombination in brome mosaic virus. Acta Biochim. Pol. 52:833-844.

Alonso, J. M., Stepanova, A. N., Leisse, T. J., Kim, C. J., Chen, H., Shinn, P., Stevenson, D. K., Zimmerman, J., Barajas, P., Cheuk, R., Gadrinab, C., Heller, C., Jeske, A., Koesema, E., Meyers, C. C., Parker, H., Prednis, L., Ansari, Y., Choy, N., Deen, H., Geralt, M., Hazari, N., Hom, E., Karnes, M., Mulholland, C., Ndubaku, R., Schmidt, I., Guzman, P., Aguilar-Henonin, L., Schmid, M., Weigel, D., Carter, D. E., Marchand, T., Risseeuw, E., Brogden, D., Zeko, A., Crosby, W. L., Berry, C. C., and Ecker. J. R. 2003. Genome-wide insertional mutagenesis of Arabidopsis thaliana. Science 301:653-657.

Baulcombe, D. C. 2005. RNA silencing. Trends Biochem. Sci. 30:290293.

Baulcombe, D. C. 2006. Short silencing RNA: The dark matter of genetics. Cold Spring Harb. Sym. Quant. Biol. 71:13-20.

Blevins, T., Rajeswaran, R., Shivaprasad, P. V., Beknazariants, D., SiAmmour, A., Park, H. S., Vazquez, F., Robertson, D., Meins, F., Jr., Hohn, T., and Pooggin, M. M. 2006. Four plant Dicers mediate viral small RNA biogenesis and DNA virus induced silencing. Nucleic Acids Res. 34:6233-6246.

Bouche, N., Lauressergues, D., Gasciolli, V., and Vaucheret, H. 2006. An antagonistic function for Arabidopsis DCL2 in development and a new function for DCL4 in generating viral siRNAs. EMBO (Eur. Mol. Biol. Organ.) J. 25:3347-3356.

Bruyere A., Wantroba, M., Flasinski, S., Dzianott, A., and Bujarski, J. J. 2000. Frequent homologous recombination events between molecules of one RNA component in a multipartite RNA virus. J. Virol. 74:42144219.

Bujarski, J. J. 2008a. Recombination. In Encyclopedia of Virology, Third Edition, Elsevier, New York.

Bujarski, J. J. 2008b. Bromoviridae. In Encyclopedia of Virology, Third Edition, Elsevier, New York.

Chao, M. 2007. RNA recombination in hepatitis delta virus: Implications regarding the abilities of mammalian RNA polymerases. Virus Res. 127:208-215.

Chapman, E. J., and Carrington, J. C. 2007. Specialization and evolution of endogenous small RNA pathways. Nat. Rev. Genet. 8:884-896.

Cheng, C. P., Serviene, E., and Nagy, P. D. 2006. Suppression of viral RNA recombination by a host exoribonuclease. J. Virol. 80:2631-2640.

Chetverin, A. B., Kopein, D. S., Chetverina, H. V., Demidenko, A. A., and Ugarov, V. I. 2005. Viral RNA-directed RNA polymerases use diverse mechanisms to promote recombination between RNA molecules. J. Biol. Chem. 280:8748-8755.

Cogoni, C., and Macino, G. 1999. Posttranscriptional gene silencing in Neurospora by a RecQ DNA helicase. Science 286:2342-2344.

Deleris, A., Gallego-Bartolome, J., Bao, J., Kasschau, K. D., Carrington, J. C., and Voinnet, O. 2006. Hierarchical action and inhibition of plant Dicer-like proteins in antiviral defense. Science 313:68-71.

Diaz-Pendon, J. A., Li, F., Li, W.-X., and Ding, S.-W. 2007. Suppression of antiviral silencing by cucumber mosaic virus $2 \mathrm{~b}$ protein in Arabidopsis is associated with drastically reduced accumulation of three classes of viral small interfering RNAs. Plant Cell 19:2053-2063.

Ding, S. W., and Voinnet, O. 2007. Antiviral immunity directed by small RNAs. Cell 130:413-426.

Donaire, L., Wang, Y., Gonzalez-Ibeas, D., Mayer, K. F., Aranda, M. A., Llave, C. 2009. Deep-sequencing of plant viral small RNAs reveals effective and widespread targeting of viral genomes. Virology 392:203214.

Duffy, S., Shackelton, L. A., and Holmes, E. C. 2008. Rates of evolutionary change in viruses: Patterns and determinants. Nat. Rev. Genet. 9:267-276.

Dunoyer, P., and Voinnet, O. 2005. The complex interplay between plant viruses and host RNA-silencing pathways. Curr. Opin. Plant Biol. 8:415-423.

Dunoyer, P., Schott, G., Himber, C., Meyer, D., Takeda, A., Carrington, J. C., and Voinnet, O. 2010. Small RNA duplexes function as mobile silencing signals between plant cells. Science 328:912-916.

Dzianott, A., and Bujarski, J. J. 2004. Infection and RNA recombination of Brome mosaic virus in Arabidopsis thaliana. Virology 318:482-492.

Dzianott, A., Rauffer-Bruyere, N., and Bujarski, J. J. 2001. Studies on functional interaction between Brome mosaic virus replicase proteins during RNA recombination, using combined mutants in vivo and in vitro. Virology 289:137-149.

Elena, S. F., Agudelo-Romero, P., Carrasco, P., Codoner, F. M., Martın, S., Torres-Barcelo, C., and Sanjuan, R. 2008. Experimental evolution of plant RNA viruses. Heredity 100:478-483.

Escarmís, C., Lázaro, E., and Manrubia, S. C. 2006. Population bottlenecks in quasispecies dynamics. Curr. Top. Microbiol. Immunol. 299:141-170.

Faehnle, C. R., and Leemor, J.-T. 2007. Argonautes confront new small RNAs. Curr. Opin. Chem. Biol. 11:569-577.

Figlerowicz, M., Nagy, P. D., and Bujarski, J .J. 1997. A mutation in the putative RNA polymerase gene inhibits nonhomologous, but not homologous, genetic recombination in an RNA virus. Proc. Natl. Acad. Sci. U.S.A. 94:2073-2078.

Fujisaki, K., Iwahashi, F., Kaido, M., Okuno, T., and Mise, K. 2009. Genetic analysis of a host determination mechanism of bromoviruses in Arabidopsis thaliana. Virus Res. 140:103-111.

Fusaro, A. F., Matthew, L., Smith, N. A., Curtin, S. J., Dedic-Hagan, J., Ellacott, G. A., Watson, J. M., Wang, M. B., Brosnan, C., and Carroll, B. J. 2006. RNA interference-inducing hairpin RNAs in plants act through the viral defence pathway. EMBO (Eur. Mol. Biol. Organ.) Rep. 7:1168-1175.

Gmyl, A. P., Korshenko, S. A., Belousov, E. V., Khitrina, E. V., and Agol, V.I. 2003. Nonreplicative homologous RNA recombination: Promiscuous joining of RNA pieces. RNA 9:1221-1231.

Gy, I., Gasciolli, V., Lauressergues, D., Morel, J.B., Gombert, J., Proux, F., Proux, C., Vaucheret, H., and Mallory, A.C. 2007. Arabidopsis FIERY1, XRN2, and XRN3 are endogenous RNA silencing suppressors. Plant Cell 19:345134-345161.

Hamilton, A. J., and Baulcombe, D. C. 1999. A species of small antisense RNA in posttranscriptional gene silencing in plants. Science 286:950952.

Hanson, M. N., Balakrishnan, M., Roques, B. P., and Bambara, R. A. 2005. Effects of donor and acceptor RNA structures on the mechanism of strand transfer by HIV-1 reverse transcriptase. J. Mol. Biol. 353:772787. 
Janda, M., French, R., and Ahlquist, P. 1987. High efficiency T7 polymerase synthesis of infectious RNA from cloned brome mosaic virus cDNA and effects of $5^{\prime}$ extensions on transcript infectivity. Virology 158:259262

Jaskiewicz, L., and Filipowicz, W. 2008. Role of Dicer in posttranscriptional RNA silencing. Curr. Top. Microbiol. Immunol. 320:77-97.

Joshua-Tor, L. 2006. The Argonautes. Cold Spring Harb. Sym. Quant. Biol. 71:67-72.

Kim, M. J., and Kao, C. 2001. Factors regulating template switch in vitro by viral RNA-dependent RNA polymerases: Implications for RNARNA recombination. Proc. Natl. Acad. Sci. U.S.A. 98:4972-4977.

Kroner, P. A., Young, B. M., and Ahlquist, P. 1990. Analysis of the role of brome mosaic virus 1a protein domains in RNA replication, using linker insertion mutagenesis. J. Virol. 64:6110-6120.

Legrand-Abravanel, F., Claudinon, J., Nicot, F., Dubois, M., ChapuyRegaud, S., Sandres-Saune, K., Pasquier, K., and Izopet, J. 2007. New natural intergenotypic (2/5) recombinant of Hepatitis C virus. J. Virol. 81:4357-4362.

Llave, C. Kasschau, K. D., and Carrington, J. C. 2000. Virus-encoded suppressor of posttranscriptional gene silencing targets a maintenance step in the silencing pathway Proc. Natl. Acad. Sci. U.S.A. 97:13401-13406.

Llave C., Kasschau, K. D., and Carrington, J. C. 2000. Virus-encoded suppressor of posttranscriptional gene silencing targets a maintenance step in the silencing pathway. Proc. Natl. Acad. Sci. U.S.A. 97:1340113406.

Molnar, A., Csorba, T., Lakatos, L., Varallyay, E., Lacomme, C., and Burgyan, J. 2005. Plant virus-derived small interfering RNAs originate predominantly from highly structured single-stranded viral RNAs. J. Virol. 79:7812-7818.

Morel, J. B., Godon, C., Mourrain, P., Beclin, C., Boutet, S., Feuerbach, F., Proux, F., and Vaucheret, H. 2002. Fertile hypomorphic ARGONAUTE (ago1) mutants impaired in posttranscriptional gene silencing and virus resistance. Plant Cell 14:629-639.

Mourrain, P., Beclin, Ch., Elmayan, T., Feuerbach, F., Godon, C., Morel, J.-B., Jouette, D., Lacombe, A.-M., Nikic, S., Picault, N., Remoue, K. Sanial, M., Vo, T.-A., and Vaucheret, H. 2000. Arabidopsis sgs2 and sgs3 genes are required for posttranscriptional gene silencing and natural virus resistance. Cell 101:533-542.

Müller, S., and Imler, J. L. 2007. Dicing with viruses: MicroRNAs as antiviral factors. Immunity 27:1-3.

Nagy, P. D., and Bujarski, J. J. 1993. Targeting the site of RNA-RNA recombination in brome mosaic virus with antisense sequences. Proc. Natl. Acad. Sci. U.S.A. 90:6390-6394.

Nagy, P. D., Dzianott, A., Ahlquist, P., and Bujarski, J. J. 1995. Mutations in the helicase-like domain of protein 1a alter the sites of RNA-RNA recombination in brome mosaic virus. J. Virol. 69:2547-2556.

Nagy, P. D., Ogiela, C., and Bujarski, J. J. 1999. Mapping sequences active in homologous RNA recombination in brome mosaic virus: Prediction of recombination hot spots. Virology 254:92-104.

Olsthoorn, R. C., Bruyere, A., Dzianott, A., and Bujarski, J. J. 2002. RNA recombination in brome mosaic virus: Effects of strand-specific stemloop inserts. J. Virol. 76:12654-12662.

Rana, T. M. 2007. Illuminating the silence: Understanding the structure and function of small RNAs. Nat. Rev. Mol. Cell Biol. 8:23-36.

Rosso, M. G., Li, Y., Strizhov, N., Reiss, B., Dekker, K., and Weisshaar, B. 2003. An Arabidopsis thaliana T-DNA mutagenized population (GABI-
Kat) for flanking sequence tag-based reverse genetics. Plant Mol. Biol. 53:247-259.

Segers, G. C., Zhang, X., Deng, F., Sun, Q., and Nuss D. L. 2007. Evidence that RNA silencing functions as an antiviral defense mechanism in fungi. PNAS 104:12902-12906.

Souret, F. F., Kastenmayer, J. P., and Green, P. J. 2004. AtXRN4 degrades mRNA in Arabidopsis and its substrates include selected miRNA targets. Mol. Cell 15:173-183.

Stram, Y., and Kuznietzova, L. 2006. Inhibition of viruses by RNA interference. Virus Genes 32:299-306.

Sun, Q., Choi, G. H., and Nuss, D. L. 2009. A single Argonaute gene is required for induction of RNA silencing antiviral defense response and promotes viral RNA recombination. Proc. Natl. Acad. Sci. U.S.A. 106:17927-17932.

Takahashi, H., Miller, J., Nozaki, Y., Takeda, M., Shah, J., Hase, S., Ikegami, M., Ehara, Y., Dinesh-Kumar, S. P., and Sukamto. 2002. RCY1, an Arabidopsis thaliana RPP8/HRT family resistance gene, conferring resistance to Cucumber mosaic virus requires salicylic acid, ethylene and a novel signal transduction mechanism. Plant $\mathbf{J}$ 32:655-667.

Urbanowicz, A., Alejska, M., Formanowicz, P., Blazewicz, J., Figlerowicz, M., and Bujarski, J. J. 2005. Homologous crossovers among molecules of Brome mosaic bromovirus RNA1 or RNA2 segments in vivo. J. Virol. 79:5732-5742.

Vaucheret, H. 2006. Post-transcriptional small RNA pathways in plants: Mechanisms and regulations. Genes Dev. 20:759-771.

Vazquez, F. 2006. Arabidopsis endogenous small RNAs: Highways and byways. Trends Plant Sci. 11:460-468.

Wang, X. B., Wu, Q., Ito, T., Cillo, F., Li, W. X., Chen, X., Yu, J. L., and Ding, S. W. 2010. RNAi-mediated viral immunity requires amplification of virus-derived siRNAs in Arabidopsis thaliana. Proc. Natl. Acad. Sci. U.S.A. 107:484-489.

White, K. A., and Nagy, P. D. 2004. Advances in the molecular biology of tombusviruses: Gene expression, genome replication, and recombination. Prog. Nucleic Acid Res. Mol. Biol. 78:187-226.

Winer, B. J. 1962. Pages 196-198 in: Statistical Principles in Experimental Design. McGraw-Hill Book Company, New York.

Xie, Z., Allen, E., Wilken, A., and Carrington, J. C. 2005. DICER-LIKE 4 functions in trans-acting small interfering RNA biogenesis and vegetative phase change in Arabidopsis thaliana. Proc. Natl. Acad. Sci. U.S.A. 102:12984-12989.

Zamore, P. D. 2004. Plant RNAi: How a viral silencing suppressor inactivates siRNA. Curr. Biol. 14:R198-200.

Zamore, P. D., and Haley, B. 2005. Ribo-genome: The big world of small RNAs. Science 309:1519-1524.

Zhang, X., and Nuss D. L. 2008. A host dicer is required for defective viral RNA production and recombinant virus vector RNA instability for a positive sense RNA virus. Proc. Natl. Acad. Sci. U.S.A. 105:16749 16754.

Zhang, X., Yuan, Y. R., Pei, Y., Lin, S. S., Tuschl, T., Patel, D. J., and Chua, N. H. 2006. Cucumber mosaic virus-encoded 2b suppressor inhibits Arabidopsis Argonaute1 cleavage activity to counter plant defense. Genes Dev. 20:3255-3268.

Zhang, X., Henderson, I. R., Lu, C., Green, P. J., and Jacobsen, S. E. 2007. Role of RNA polymerase IV in plant small RNA metabolism. Proc. Natl. Acad. Sci. U.S.A. 104:4536-4541. 